\title{
Prevalencia y caracterización de los factores asociados a dispareunia en mujeres con antecedente de parto vaginal o cesárea
}

\section{Prevalence and characterization of factors associated with dyspareunia in women with a history of vaginal delivery or cesarean section}

\author{
Franklin J. Espitia-De La Hoz* \\ Clínica Sexológica, Hathor, Armenia, Quindío, Colombia
}

\section{Resumen}

Introducción: El deterioro de la función sexual asociado a la dispareunia después del parto es una importante preocupación para muchas mujeres. Objetivo: Establecer la prevalencia y caracterizar los factores asociados a la dispareunia en mujeres con antecedente de parto vaginal o cesárea. Método: Estudio de corte transversal realizado en 975 mujeres mayores de 18 años, residentes en Armenia (Colombia), con 6 meses o más en posparto (vaginal o cesárea), sexualmente activas, entre 2013 y 2017. Se utilizó como instrumento el Índice de Función Sexual Femenino Abreviado (IFSFA-6). Se midieron variables sociodemográficas y obstétricas relacionadas con la dispareunia. Se hizo estadística descriptiva. Los factores asociados se evaluaron comparando los dos grupos mediante odds ratio (OR) e intervalo de confianza del 95\% (IC95\%). Resultados: La edad promedio fue de 27,12 \pm 4,48 años. La prevalencia de dispareunia fue del 35,69\%. Los factores de riesgo más involucrados fueron la episiotomía (OR: 1,58; IC95\%: 1,29-2,15; $p=0,003$ ), el parto instrumentado (OR: 1,91; IC95\%: 1,31-3,17; $p=0,027)$, haber tenido tres o más partos vaginales (OR: 1,85; IC95\%: 1,42-2,46; $p<0,001)$ ] y haber tenido dos o más cesáreas (OR: 1,64; IC95\%: 1,27-2,18; $p$ < 0,001). Las mujeres con dispareunia tienen menos encuentros sexuales semanales (2; rango: $0-3)$ que las mujeres sin dispareunia (5; rango: $2-7)(p=0,003)$. Conclusiones: La prevalencia de dispareunia en el posparto, en las mujeres de Armenia, sobrepasa un tercio de la población. Se hace necesario promover programas preventivos, dirigidos a los profesionales de la salud que asisten a la mujer durante el parto, acerca del análisis de la verdadera necesidad de la episiotomía o la cesárea.

Palabras clave: Dispareunia. Cesárea. Coito. Epidemiología. Posparto. Mujeres.

\section{Abstract}

Introduction: The deterioration of sexual function, associated with dyspareunia after childbirth, is an important concern for many women. Objective: To establish the prevalence and characterize the factors associated with dyspareunia in women, with a history of vaginal delivery or cesarean section. Method: Cross-sectional study, carried out in 975 sexually active women older than 18 years, residents in Armenia (Colombia), with 6 or more months postpartum (vaginal deliveries and cesarean sections); between 2013 and 2017. The Abbreviated Female Sexual Function Index (IFSFA-6) was used as an instrument. Sociodemographic and obstetric variables related to dyspareunia were measured. Descriptive statistics were made. Associated factors were evaluated comparing the two groups using odds ratio (OR) and 95\% confidence interval (95\% Cl). Results: The average age was $27.12 \pm 4.48$ years. The prevalence of dyspareunia was $35.69 \%$. The risk factors most involved were:

Disponible en internet: 23-12-2021 Rev Chil Obstet Ginecol. 2021;86(5):435-443

www. rechog.com CC BY-NC-ND (https://creativecommons.org/licenses/by-nc-nd/4.0/). 
Rev Chil Obstet Ginecol. 2021;86(5)

episiotomy (OR: 1.58; 95\%Cl: 1.29-2.15; $p=0.003$ ), instrumented delivery (OR: 1.91; 95\%Cl: 1.31-3.17; $p=0.027$ ), three or more vaginal deliveries (OR: 1.85 ; $95 \% \mathrm{Cl}: 1.42-2.46 ; p<0.001$ ) and two or more caesarean sections (OR: 1.64; $95 \% \mathrm{Cl}$ : 1.272.18; $p<0.001$ ). Women with dyspareunia have fewer weekly sexual encounters (2; range: 0-3) than women without dyspareunia (5; range: $2-7)(p=0.003)$. Conclusions: The prevalence of dyspareunia in postpartum, in Armenian women, exceeds $1 / 3$ of the population. It is necessary to promote preventive programs, aimed at health professionals who assist women during childbirth, regarding the analysis of the true need for episiotomy or cesarean section.

Key words: Dyspareunia. Cesarean section. Coitus. Epidemiology. Postpartum. Women.

\section{Introducción}

La dispareunia en las mujeres se define como el dolor recurrente o persistente con la actividad sexual, que causa marcada angustia o conflicto interpersonal'. En el posparto es un frecuente padecimiento, en especial secundario a problemas de origen obstétrico y ginecológico².

La incidencia de relaciones sexuales dolorosas varía entre el $8 \%$ y el $22 \% 3,4$. En un estudio de prevalencia que involucró a 3017 mujeres mostró una incidencia máxima del $4,3 \%$ en el grupo de edad de 20 a 29 años ${ }^{5}$, con menor porcentaje para cada década subsecuente. No obstante, las principales causas de dispareunia varían según el grupo etario ${ }^{6-8}$.

Los factores de riesgo más asociados a la aparición de dispareunia incluyen edad muy joven, nivel de educación inferior a título universitario, síntomas del tracto urinario, mala salud, problemas emocionales o estrés, disminución de ingresos ${ }^{1,3}$, etc. En relación al parto, se asocia con el trauma perineal (episiotomía, laceraciones y desgarros en el canal del parto o parto instrumentado) $)^{9,10}$.

La dispareunia involucra muchos aspectos de la vida de la mujer. Es tan catastrófica que puede derivar en insatisfacción sexual, y si ya existía antes, es mucho lo que empeora ${ }^{11}$, llevando al deterioro de su calidad de vida, ya que suele afectar negativamente la relación de pareja o la autoestima?.

En el estudio de la dispareunia es esencial tanto una adecuada exploración física como el uso de cuestionarios, entre los que sobresale el Índice de Función Sexual Femenino, versión abreviada (IFFS-6). Es una herramienta corta y fácil de usar, la cual incluye seis ítems $^{12}$. Está basada en el Female Sexual Function Index $(\mathrm{FSFI})^{13}$, instrumento creado para ayudar en el diagnóstico de las disfunciones sexuales (al explorar subjetivamente las dimensiones involucradas en el funcionamiento sexual femenino), que puede discriminar entre mujeres con riesgo de disfunción sexual de otras sin riesgo de presentarla. Los efectos del IFFS-6 parecen ser paralelos a los resultados obtenidos con la versión original, pero con un tiempo de detección más optimizado $^{12}$.

Es incuestionable el carácter multifactorial y multisistémico de la aparición de la dispareunia ${ }^{4,7}$, e indudablemente de la incidencia de su aparición posparto, y son muchos los factores asociados factibles de ser controlados por el obstetra. Por ello, el objetivo del presente estudio consistió en establecer la prevalencia y caracterizar los factores asociados a la dispareunia en mujeres en edad reproductiva con antecedente de parto vaginal o cesárea, en tres clínicas privadas de Armenia, Quindío (Colombia).

\section{Método}

\section{Diseño y población}

Estudio de corte transversal en el que se incluyeron 975 mujeres mayores de 18 años, residentes en Armenia, con 6 meses o más en posparto (partos vaginales y cesáreas), que no estuvieran amamantando y sexualmente activas en los últimos 3 meses, que asistieron por primera vez al Programa de Anticoncepción y Planificación Familiar en tres clínicas privadas de Armenia (Quindío), Colombia, entre el 1 de marzo de 2013 y el 31 de agosto de 2017. Se excluyeron las analfabetas, aquellas con parto gemelar, prolapso genital o antecedente de cáncer, las que no tenían todas las variables del estudio y las que no firmaron el consentimiento informado. El tamaño de la muestra se calculó en 24.375 mujeres participantes, con base en una prevalencia estimada del $42 \%$, un error de muestreo del $5 \%$ y un nivel de confianza del $95 \%$, obteniéndose un tamaño de muestra mínimo de 420 mujeres. Se realizó un muestreo aleatorio simple usando una tabla de números aleatorios.

\section{Procedimiento}

Las mujeres que refirieron dolor coital, de manera espontánea, fueron evaluadas por un médico general diplomado en sexología, quien determinaba si 
cumplían con los criterios de elegibilidad del estudio; en su defecto, las pacientes fueron identificadas a partir de la base de datos proporcionada por las clínicas participantes. Para esto se utilizó el código N94.1 Dispareunia de la Clasificación Internacional de Enfermedades 10 a revisión (CIE-10). En su totalidad, las participantes fueron evaluadas por el investigador principal (especialista en sexología clínica).

Si la paciente cumplía con los criterios de selección, una enfermera profesional, que era parte del equipo de investigación, se encargó de invitarla a participar de manera voluntaria, informándole de los objetivos del estudio, así como de los procedimientos que se realizarían, y solicitándole la firma del consentimiento informado. Luego de la firma del consentimiento, una auxiliar le entregaba el IFFS-6 y le enseñaba el correcto diligenciamiento, de manera individual y en un ambiente privado, además de completar el interrogatorio de las otras variables del estudio.

El instrumento utilizado fue el IFFS- $6^{12}$, el cual es un cuestionario autoadministrado, útil para identificar la presencia disfunciones sexuales en las mujeres. Este instrumento se compone de seis preguntas derivadas del FSFI-19 original $^{13}$, cada una de las cuales cubre uno de los dominios originales: deseo (ítem original \# 2), excitación (ítem original \# 4), lubricación (ítem original \# 7), orgasmo (ítem original \# 11), satisfacción (ítem original \# 16) y dolor (ítem original \# 17). Cada pregunta proporciona una puntuación que varía de 0 a 5 , y la sumatoria de estos dominios arroja un puntaje total final para el cuestionario. Cuanto más alto es el puntaje, mejor es la sexualidad. Se ha propuesto un valor de corte de 19 o menos para identificar a las mujeres en riesgo de disfunción sexual ${ }^{12}$. El cuestionario cuenta con la pregunta 6 , que es una subescala para dolor sexual (dispareunia).

EI IFFS-6 ha demostrado ser una versión válida y fiable, ya que tiene una sensibilidad de 0,93 y una especificidad de 0,94, con valor de $p<0,001$ y una alta consistencia interna (alfa de Cronbach $=0,78)^{12}$. Este cuestionario no está validado en la población colombiana, pero se escogió como instrumento del estudio por haber sido validado en español en una población de mujeres ecuatorianas ${ }^{14}$.

Se organizó un grupo de trabajo en cada institución participante para recoger la información. Cada grupo estuvo conformado por tres auxiliares de enfermería y una enfermera profesional, especialista en auditoría en salud, que identificaban las historias clínicas de las pacientes de la población del estudio. Se tomó información de las variables sociodemográficas, clínicas basales, tipo de parto y complicaciones del parto. Todas las enfermeras estaban entrenadas en el diligenciamiento del IFSF-6 y eran conocedoras de los propósitos del estudio. Los tres grupos estaban liderados por el autor principal de la investigación.

En su totalidad, las mujeres fueron atendidas por el médico especialista en sexología, quien hacía la evaluación y la valoración de las eventuales causas de la dispareunia, encargándose del respectivo manejo.

\section{Variables medidas}

Se consideraron variables sociodemográficas (edad, raza, estado civil, ocupación, nivel educativo, área de residencia, estrato socioeconómico y régimen de afiliación al Sistema General de Seguridad Social en Colombia), hábitos (consumo de alcohol, tabaquismo y uso de sustancias psicoactivas), antecedentes gineco-obstétricos, antecedente de infecciones de transmisión sexual (ITS), antecedentes quirúrgicos (cirugía abdomino-pélvica), antecedente de violación o abuso sexual, edad de inicio de la vida sexual, edad del primer embarazo y antecedente de dispareunia. Además, se interrogó sobre orientación sexual, antecedente de dispareunia, número de compañeros sexuales, frecuencia coital semanal, masturbación, uso de lubricante y afectación de la relación de pareja por la dispareunia.

La información se tabuló y archivó en un formulario de Excel 15.0, especialmente diseñado para esta investigación.

\section{Análisis estadístico}

En el análisis de los datos se utilizó estadística descriptiva. Para las variables cuantitativas se calculó la media con la desviación estándar, la mediana y el rango, y para las variables cualitativas se realizaron tablas de frecuencias. Se llevaron a cabo un análisis univariado para determinar la prevalencia de la dispareunia y un análisis bivariado para relacionar la presencia de dispareunia con las características sociodemográficas y los factores asociados, mediante las pruebas $\chi^{2}$, $\mathrm{t}$ de Student, $\mathrm{U}$ de Mann-Whitney y exacta de Fisher, de acuerdo con la distribución de las variables, con una significación estadística del $5 \%$. Los factores asociados se evaluaron comparando los dos grupos por medio de odds ratio (OR) e intervalo de confianza del 95\% (IC95\%). Para el procesamiento de datos se utilizó el paquete estadístico STATA versión 14.0 


\section{Aspectos éticos}

La investigación fue aprobada por el Comité de Ética Médica de cada una de las clínicas participantes y cumplió los principios éticos de investigación médica en seres humanos establecidos en la Declaración de Helsinki y en la Resolución 8430 de 1993 del Ministerio de Salud de Colombia, por la cual se establecen las normas científicas, técnicas y administrativas para investigación en salud. Se garantizó y mantuvo la confidencialidad de la información para proteger los derechos de las participantes en el estudio. Todas las pacientes firmaron el consentimiento informado.

\section{Resultados}

El análisis se hizo con 975 participantes, equivalentes al $71,84 \%$ de la población seleccionada, con una edad media de 27,12 $\pm 4,48$ años. El 72,61\% pertenecían al régimen contributivo del Sistema General de Seguridad Social en Salud y el 58,87\% eran de origen urbano. El 50,97\% ( $n=497 / 975)$ eran de estrato socioeconómico medio. La distribución de las características sociodemográficas se detalla en la tabla 1.

En la población de 975 mujeres de este estudio, en $407(41,74 \%)$ se resolvió su último embarazo por la vía de la cesárea y en $568(58,25 \%)$ se asistió al parto por vía vaginal, de las cuales el $49,11 \%(n=279 / 568)$ se sometieron a episiotomía y el $4,75 \%(n=27 / 568)$ fueron partos instrumentados con espátulas $(2,99 \%)$ o con fórceps $(1,76 \%)$. Las características obstétricas se detallan en la tabla 2.

Respecto de los hábitos, el 72,92\% ( $n=711 / 975)$ refirió consumir alcohol de manera regular (4-5 veces por mes), el tabaquismo se encontró en el $19,28 \%$ de las participantes $(n=188 / 975)$, y el $5,94 \%(n=58 / 975)$ usaban sustancias psicoactivas.

Entre los antecedentes quirúrgicos destacan apendicectomía $(16,82 \%)$, esterilización tubárica $(14,56 \%)$, colecistectomía $(11,17 \%)$ y salpingectomía unilateral por embarazo ectópico (3,89\%).

La edad media de la menarquia fue de 13,75 $\pm 1,94$ años (límite inferior: 9 años; límite superior: 21 años). La edad promedio de inicio de la vida sexual fue de $15,78 \pm 1,64$ años. La edad media del primer embarazo fue de $16,92 \pm 1,73$ años. El $53,12 \%$ tenía dos o más hijos. El antecedente de ITS en la población general arrojó una frecuencia del 20,1\% ( $n=196 / 975)$. El antecedente de violación o abuso sexual (en algún momento de la vida) estuvo presente en el 9,94\% ( $n=97 / 975)$ de la población total de participantes.
Tabla 1. Distribución de las características sociodemográficas en mujeres con dispareunia posparto. Armenia, Colombia, 2013 a 2017

\begin{tabular}{|c|c|c|c|}
\hline & $\begin{array}{l}\text { Con } \\
\text { dispareunia } \\
n=348\end{array}$ & 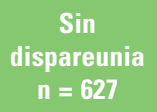 & p \\
\hline Edad, media \pm DE (años) & $26,73 \pm 4,14$ & $27,51 \pm 4,83$ & 0,741 \\
\hline Peso, media $\pm \mathrm{DE}(\mathrm{kg})$ & $57,81 \pm 5,25$ & $53,76 \pm 4,35$ & 0,361 \\
\hline Talla, media $\pm \mathrm{DE}(\mathrm{m})$ & $1,58 \pm 0,76$ & $1,59 \pm 0,84$ & 0,582 \\
\hline IMC, media $\pm \mathrm{DE}$ & $22,38 \pm 3,51$ & $21,09 \pm 2,37$ & 0,627 \\
\hline $\begin{array}{l}\text { Raza } \\
\text { Hispánicas, n (\%) } \\
\text { Afrocolombianas, n (\%) } \\
\text { Indígenas, n (\%) }\end{array}$ & $\begin{array}{c}236(67,81) \\
91(26,14) \\
21(6,03)\end{array}$ & $\begin{array}{c}472(75,27) \\
135(21,53) \\
20(3,18)\end{array}$ & $\begin{array}{l}0,372 \\
0,432 \\
0,649\end{array}$ \\
\hline $\begin{array}{l}\text { Estado civil } \\
\text { Casadas o con pareja } \\
\text { estable, n (\%) } \\
\text { Solteras, n (\%) } \\
\text { Divorciadas, n (\%) }\end{array}$ & $\begin{array}{l}267(76,72) \\
43(12,35) \\
38(10,91)\end{array}$ & $\begin{array}{c}486(77,51) \\
62(9,88) \\
79(12,59)\end{array}$ & $\begin{array}{l}0,375 \\
0,495 \\
0,579\end{array}$ \\
\hline $\begin{array}{l}\text { Nivel de educación } \\
\text { Primaria, } \mathrm{n}(\%) \\
\text { Secundaria, n (\%) } \\
\text { Técnica, n (\%) } \\
\text { Profesionales, n (\%) }\end{array}$ & $\begin{array}{c}25(7,18) \\
83(23,85) \\
101(29,02) \\
139(39,94)\end{array}$ & $\begin{array}{l}61(9,72) \\
157(25,03) \\
146(23,28) \\
263(41,94)\end{array}$ & $\begin{array}{l}0,474 \\
0,813 \\
0,312 \\
0,729\end{array}$ \\
\hline $\begin{array}{l}\text { Ocupación } \\
\text { Amas de casa, n (\%) } \\
\text { Empleadas, n (\%) } \\
\text { Desempleadas, n (\%) }\end{array}$ & $\begin{array}{c}202(58,04) \\
65(18,67) \\
81(23,27)\end{array}$ & $\begin{array}{l}372(59,33) \\
119(18,97) \\
136(21,69)\end{array}$ & $\begin{array}{l}0,381 \\
0,729 \\
0,684\end{array}$ \\
\hline
\end{tabular}

DE: desviación estándar; IMC: índice de masa corporal.

Tabla 2. Características obstétricas en mujeres con dispareunia posparto. Armenia, Colombia, 2013 a 2017

\begin{tabular}{l|c|c|c} 
& $\begin{array}{c}\text { Con dispareunia } \\
n=348\end{array}$ & $\begin{array}{c}\text { Sin } \\
\text { dispareunia } \\
n=627\end{array}$ & 0,039 \\
$\begin{array}{l}\text { Paridad, } \\
\text { mediana (rango) }\end{array}$ & $3(1-6)$ & $3(1-7)$ & 0,732 \\
$\begin{array}{l}\text { Parto vaginal, } \\
\text { mediana (rango) }\end{array}$ & $2(1-6)$ & $2(1-7)$ & 0,048 \\
$\begin{array}{l}\text { Cesárea, } \\
\text { mediana (rango) }\end{array}$ & $2(1-6)$ & $1(1-5)$ & $<0,001$ \\
$\begin{array}{l}\text { Episiotomía, } \mathrm{n} \\
\text { (\%) }\end{array}$ & $219(62,93)$ & $60(9,56)$ & \\
$\begin{array}{l}\text { Parto } \\
\text { instrumentado, } \\
\mathrm{n}(\%)\end{array}$ & $22(6,32)$ & $5(0,79)$ & $<0,001$ \\
\end{tabular}

DE: desviación estándar.

La orientación sexual fue de predominio heterosexual, en el $87,28 \%(n=851 / 975)$. El número de compañeros sexuales arrojó una mediana de 7 (rango: 1 a 
Tabla 3. Índice de Función Sexual Femenino, versión abreviada (IFFS-6), en mujeres con dispareunia posparto. Armenia, Colombia, 2013 a 2017

\begin{tabular}{|c|c|c|c|c|c|c|}
\hline \multirow[t]{2}{*}{ Dominio } & \multirow[t]{2}{*}{ Media } & \multicolumn{2}{|c|}{ IC95\% } & \multirow[t]{2}{*}{ DE } & \multirow[t]{2}{*}{ Mínimo } & \multirow[t]{2}{*}{ Máximo } \\
\hline & & Límite inferior & Límite superior & & & \\
\hline Deseo & 3,71 & 3,52 & 3,94 & 0,82 & 1,2 & 6,0 \\
\hline Excitación & 3,82 & 3,61 & 4,01 & 0,89 & 0,0 & 6,0 \\
\hline Lubricación & 3,65 & 3,68 & 4,06 & 1,12 & 0,0 & 6,0 \\
\hline Orgasmo & 3,79 & 3,75 & 4,59 & 1,31 & 0,0 & 6,0 \\
\hline Satisfacción & 3,68 & 3,49 & 4,08 & 1,43 & 0,8 & 6,0 \\
\hline Dolor & 4,82 & 4,47 & 4,95 & 0,94 & 0,0 & 6,0 \\
\hline Total & 23,47 & & & 1,08 & & \\
\hline
\end{tabular}

DE: desviación estándar; IC95\%: intervalo de confianza del 95\%.

$>15)$. La frecuencia coital semanal reportó una mediana de 4 (rango: 0-5).

El antecedente de dispareunia se observó en el $6,35 \%(n=62 / 975)$. El uso de lubricante era una práctica común en el 20,82\% ( $n=203 / 975)$ de las participantes. La masturbación era una práctica frecuente en el $35,79 \%(n=349 / 975)$.

La puntuación global del IFFS-6 fue de 23,47 \pm 5,71 puntos, mientras que en las mujeres con dispareunia fue de $17,51 \pm 6,29$ puntos. El dominio sexual más deteriorado fue el dolor (incomodidad para la penetración durante el coito), con una puntuación media de $4,82 \pm 0,94$. En la tabla 3 se detallan los dominios del IFFS-6.

La prevalencia de dispareunia en esta población de mujeres fue del 35,69\% ( $n=348 / 975)$. La prevalencia fue mayor en las mujeres afroamericanas, con un $41,09 \%$ ( $n=143 / 348)$, que en las indígenas, con un $4,31 \%(p<0,05)$. El 18,67\% ( $n=65 / 348)$ reportaron afectación de la relación de pareja a causa de la dispareunia.

Se encontró que las mujeres a quienes se instrumentó el parto presentaron mayor frecuencia de dispareunia que las que tuvieron partos no instrumentados $(45,45 \%$ frente a $20 \% ; p=0,036)$. Así mismo, la realización de episiotomía se asoció con mayor frecuencia de dispareunia $(44,91 \%$ frente a $25,14 \%$; $p<0,001)$, y de igual modo, en las mujeres a las que se hizo cesárea se encontró mayor frecuencia de dispareunia en comparación con las que no tuvieron cesárea $(42,22 \%$ frente a $24,44 \% ; p=0,039$ ), observándose una mayor prevalencia entre las que tenían dos o más cesáreas respecto a las que solo tenían una $(33,76 \%$ frente a $8,46 \% ; p=0,0027$ ).

La presencia de dispareunia fue más frecuente en las mujeres que no practicaban la masturbación $(31,78 \%)$, en las que fumaban $(27,13 \%)$, en las que completaron tres o más partos vaginales $(24,03 \%)$, en las que tenían inicio de vida sexual antes de los 18 años $(22,48 \%)$, en aquellas con antecedente de embarazo antes de los 21 años (20,15\%), en las que habían tenido tres o más parejas sexuales $(19,37 \%)$, en aquellas con antecedente de ITS (17,05\%), en las consumidoras de alcohol $(16,59 \%)$ y en las que habían sufrido violación o abuso sexual (14,63\%).

Se observó que la dispareunia fue menos frecuente en las mujeres con un solo parto (14,72\%), en las que iniciaron sus relaciones sexuales después de los 18 años $(13,17 \%)$, en las que solo habían tenido un compañero sexual $(10,85 \%)$ y en las que no habían sido sometidas a episiotomía (10,07\%), mientras que las mujeres multíparas tuvieron mayor riesgo de presentar dispareunia (OR: 2,04; IC 95\%: 1,32-3,27) y también aquellas con antecedente de dos 0 más cesáreas (OR: 1,64; IC 95\%: 1,27-2,18) ( $p<0,001)$. En la tabla 4 se detallan las variables asociadas a la prevalencia de dispareunia.

El antecedente de violación o abuso sexual estuvo presente en el $6,53 \%(n=41 / 627)$ de las mujeres $\sin$ dispareunia, mientras que en la población de mujeres con dispareunia dicho antecedente fue del $35,34 \%$ $(n=123 / 348)$.

Al contrastar la prevalencia de dispareunia según la edad, se observó que alrededor de la mitad de las mujeres con dispareunia tenían una edad promedio de 
Tabla 4. Factores asociados a dispareunia en mujeres con dispareunia posparto. Armenia, Colombia, 2013 a 2017

\begin{tabular}{|c|c|c|c|c|c|}
\hline Variables & $\begin{array}{l}\text { Con dispareunia } \\
n=348(\%)\end{array}$ & $\begin{array}{c}\text { Sin dispareunia } \\
n=627(\%)\end{array}$ & OR & IC95\% & p \\
\hline $\begin{array}{c}\text { Edad de ir } \\
\begin{array}{l}10-14 \\
15-17 \\
18-20 \\
>21\end{array}\end{array}$ & $\begin{array}{c}59(16,95 \%) \\
207(59,48 \%) \\
62(17,81 \%) \\
20(5,74 \%)\end{array}$ & $\begin{array}{c}113(18,02 \%) \\
369(58,85 \%) \\
112(17,86 \%) \\
33(5,26 \%)\end{array}$ & $\begin{array}{l}1,65 \\
1,62 \\
1,21 \\
1,02\end{array}$ & $0,93-2,97$ & 0,003 \\
\hline $\begin{array}{c}\text { Edad de } p \\
10-14 \\
15-17 \\
18-20 \\
>21\end{array}$ & $\begin{array}{c}41(11,78 \%) \\
212(60,91 \%) \\
83(23,85 \%) \\
12(3,44 \%)\end{array}$ & $\begin{array}{c}61(9,72 \%) \\
355(56,61 \%) \\
125(19,93 \%) \\
86(13,71 \%)\end{array}$ & $\begin{array}{l}2,16 \\
2,07 \\
1,56 \\
1,35\end{array}$ & $1,32-3,48$ & $<0,001$ \\
\hline $\begin{array}{l}\text { Episiotomí } \\
\text { Sí } \\
\text { No }\end{array}$ & $\begin{array}{l}218(62,64 \%) \\
130(37,35 \%)\end{array}$ & $\begin{array}{c}61(9,72 \%) \\
566(90,27 \%)\end{array}$ & $\begin{array}{l}1,58 \\
1,08\end{array}$ & $1,29-2,15$ & 0,006 \\
\hline $\begin{array}{l}\text { Anteceder } \\
\text { Sí } \\
\text { No }\end{array}$ & $\begin{array}{c}22(6,32 \%) \\
326(93,67 \%)\end{array}$ & $\begin{array}{c}5(0,79 \%) \\
622(99,20 \%)\end{array}$ & $\begin{array}{l}1,91 \\
1,02\end{array}$ & $1,31-3,17$ & 0,027 \\
\hline $\begin{array}{l}\text { Anteceder } \\
\text { Sí } \\
\text { No }\end{array}$ & $\begin{array}{l}122(35,05 \%) \\
226(64,94 \%)\end{array}$ & $\begin{array}{l}268(42,74 \%) \\
359(57,25 \%)\end{array}$ & $\begin{array}{l}1,85 \\
1,07\end{array}$ & $1,42-2,46$ & $<0,001$ \\
\hline $\begin{array}{l}\text { Anteceder } \\
\text { Sí } \\
\text { No }\end{array}$ & $\begin{array}{l}235(67,52 \%) \\
113(32,47 \%)\end{array}$ & $\begin{array}{l}344(54,86 \%) \\
283(45,13 \%)\end{array}$ & $\begin{array}{l}1,64 \\
1,05\end{array}$ & 1,27-2,18 & $<0,001$ \\
\hline $\begin{array}{l}\text { Tres o má } \\
\text { Sí } \\
\text { No }\end{array}$ & $\begin{array}{c}294(84,48 \%) \\
54(15,51 \%)\end{array}$ & $\begin{array}{c}545(86,92 \%) \\
82(13,07 \%)\end{array}$ & $\begin{array}{l}1,53 \\
1,02\end{array}$ & $0,75-3,06$ & 0,009 \\
\hline $\begin{array}{l}\text { Consumo } \\
\text { Sí } \\
\text { No }\end{array}$ & $\begin{array}{c}288(82,75 \%) \\
60(17,24 \%)\end{array}$ & $\begin{array}{l}423(67,46 \%) \\
204(32,53 \%)\end{array}$ & $\begin{array}{l}1,83 \\
1,06\end{array}$ & $0,87-3,72$ & 0,003 \\
\hline $\begin{array}{l}\text { Tabaquism } \\
\text { Sí } \\
\text { No }\end{array}$ & $\begin{array}{l}288(82,75 \%) \\
112(32,18 \%)\end{array}$ & $\begin{array}{l}337(53,74 \%) \\
290(46,25 \%)\end{array}$ & $\begin{array}{l}1,83 \\
1,06\end{array}$ & $0,87-3,72$ & 0,003 \\
\hline $\begin{array}{l}\text { Anteceder } \\
\text { Sí } \\
\text { No }\end{array}$ & $\begin{array}{c}72(20,68 \%) \\
276(79,31 \%)\end{array}$ & $\begin{array}{l}124(19,77 \%) \\
503(80,22 \%)\end{array}$ & $\begin{array}{l}1,29 \\
1,03\end{array}$ & $0,66-2,64$ & 0,005 \\
\hline $\begin{array}{l}\text { Anteceder } \\
\text { Sí } \\
\text { No }\end{array}$ & $\begin{array}{c}41(11,78 \%) \\
307(88,21 \%)\end{array}$ & $\begin{array}{c}56(8,93 \%) \\
571(91,06 \%)\end{array}$ & $\begin{array}{l}1,44 \\
1,04\end{array}$ & $0,48-4,17$ & 0,003 \\
\hline
\end{tabular}

$26,43 \pm 5,37$ años, frente a $30,15 \pm 1,83$ años las que no la tenían. La proporción de dispareunia incapacitante fue reportada con mayor frecuencia en las mayores de 30 años (31-35 años) (39,36\%; $n=137 / 348)$.

En relación al nivel educativo, la mayor proporción de dispareunia se reportó en mujeres que tenían estudios de secundaria incompleta 0 menos $(42,81 \%$; $n=149 / 348$ ), mientras que la menor proporción se observó en las mujeres que estaban cursando o habían finalizado estudios universitarios $(9,19 \% ; n=32 / 348)$.
Se encontró que las mujeres que rechazaban la masturbación presentaron mayor frecuencia de dispareunia (42,63\% frente a $22,48 \% ; p=0,036)$; así mismo, el estar inconforme con su sexualidad se asoció con mayor frecuencia de dispareunia $(47,28 \%$ frente a $28,68 \% ; p<0,001$ ).

Según el estrato socioeconómico, la mayor frecuencia de dispareunia se encontró en mujeres de estrato medio o bajo $(58,33 \% ; n=203 / 348)$, y la menor frecuencia en el estrato alto $(7,47 \% ; n=26 / 348)$. 
En cuanto a la relación de pareja, se observó que las mujeres con mala armonía de pareja presentaban un riesgo mayor de dispareunia en comparación con las mujeres con mejor armonía de pareja, y el riesgo aumenta a medida que empeora la pérdida de la armonía (OR: 1,08; IC95\%: 1,12-3,65; $p=0,001$ ).

No se observaron diferencias significativas por la orientación sexual entre las heterosexuales y las homosexuales o bisexuales; sin embargo, la proporción de mujeres con dispareunia fue superior entre las heterosexuales, con un chance de 1,32 (IC95\%: 1,01-3,17; $p<0,05)$.

En cuanto a la mediana del número de encuentros sexuales coitales semanales, con frecuencia en los últimos 7 días previos a la entrevista, se encontraron diferencias significativas: las mujeres con dispareunia tenían menos encuentros sexuales semanales (2; rango: $0-3$ ) que las mujeres sin dispareunia (5; rango: $2-7)(p=0,003)$.

Aproximadamente la mitad de las mujeres con dispareunia manifestaron haber abandonado o disminuido su actividad sexual debido al dolor que tienen que soportar durante el coito, presentando diferencias significativas en comparación con el grupo de mujeres sin dispareunia (17,84\%; IC95\%: 14,28-24,57; $p=0,003$ ), constituyéndose en un factor de riesgo importante de abandono del coito.

Las tres cuartas partes de las mujeres con dispareunia consideran que el coito siempre requiere el uso de lubricantes, mientras que la mayoría de las mujeres sin dispareunia consideran que el uso de lubricantes no es necesario, presentando diferencias significativas entre los grupos $(p=0,006)$.

El 65,51\% ( $n=228 / 348)$ de las mujeres con dispareunia valoran su sexualidad como regular 0 mala, mientras que una proporción similar $(66,09 \%)$ de las mujeres sin dispareunia la valoran como excelente o buena, presentando diferencias significativas entre los grupos $(p=0,003)$.

En el análisis multivariado se mantuvieron como factores de riesgo para presentar dispareunia el antecedente de parto instrumentado (OR: 1,91; IC95\%: 1,31-3,17), la episiotomía (OR: 1,58; IC95\%: 1,29-2,15), el antecedente de dos o más cesáreas (OR: 1,64; IC95\%: 1,27-2,18) y el antecedente de tres o más partos vaginales (OR: 1,85; IC95\%: 1,42-2,46) $(p<0,05)$.

\section{Discusión}

La prevalencia de dispareunia oscila entre el 1,4\% y el $22 \%{ }^{15,16}$, porcentaje que se incrementa hasta el $17 \%$ al $36 \%$ a los 6 meses posparto ${ }^{8,16}$; sin embargo, en un estudio realizado en Turquía con 530 mujeres en posparto que habían dado a luz durante los 2 a 12 meses anteriores, Alp Yilmaz, et al. ${ }^{17}$ reportaron que el $74,3 \%$ experimentaron disfunciones sexuales, con una prevalencia de dispareunia del 50,2\%. Por su parte, Mitchell, et al..$^{18}$ estimaron la prevalencia de la dispareunia en mujeres de Gran Bretaña, en una muestra de 6669 mujeres sexualmente activas, y encontraron que el 7,5\% (IC95\%: 6,7-8,3) informaron relaciones sexuales dolorosas, de las cuales una cuarta parte experimentó síntomas con mucha frecuencia o siempre, durante $\geq$ 6 meses, que eran causa de angustia.

La prevalencia de dispareunia en el presente estudio (en mujeres con 6 meses o más tras el parto y que no estuvieran amamantando) arrojó un porcentaje cercano a la tercera parte de la población, aunque es inferior al $62 \%$ descrito por Barrett, et al..$^{19}$ en una población de 796 primíparas en un hospital universitario de Londres; sin embargo, es superior al $24 \%$ reportado por McDonald, et al. ${ }^{20}$ en Melbourne (Australia).

En la presente investigación se observó una clara influencia de la episiotomía y de la cesárea en la aparición de dispareunia posparto, lo cual es concordante con el $49 \%$ reportado por Buhling, et al. ${ }^{21}$ en Berlín, y con el 37,1\% informado por Manresa, et al. ${ }^{22}$ a los 6 meses del parto. La paridad se presenta como un importante factor asociado a dispareunia, de tal manera que el dolor coital es más frecuente en las mujeres con tres o más partos y con dos o más cesáreas (OR: 1,85, IC95\%: 1,42-2,46, frente a OR: 1,64, IC95\%: 1,27-2,18; $p<0,001)$.

De acuerdo con los hallazgos de la influencia negativa del parto instrumentado y del número de cesáreas, estos datos en su conjunto llevan a reflexionar acerca del hecho de que las alteraciones de la actividad sexual posparto son más bien parte de un proceso de inadecuada elección de los recursos de la atención del parto. En esto nos alineamos con Manresa, et al. ${ }^{22}$, quienes afirman que, en caso de traumatismo perineal o episiotomía, las probabilidades de dolor a los 10 días y de dispareunia a los 6 meses posparto son cuatro y cinco veces mayores, respectivamente.

Respecto al antecedente de ITS, la actividad sexual fue menos frecuente con respecto a las mujeres que no tenían dicho antecedente. Además, haber tenido tres o más parejas sexuales también se asoció con una mayor incidencia de dolor coital, así como el antecedente de abuso sexual. Estos datos coinciden con los reportados por otros autores ${ }^{23,24}$. 
La dispareunia fue menor en las universitarias que en las que solo cursaron la primaria o no terminaron la secundaria, lo cual apoya la afirmación de que tener solo educación primaria ${ }^{25}$ o secundaria ${ }^{26,27}$ es un factor de riesgo significativo para presentar disfunción sexual, datos coincidentes con los resultados de este estudio.

Las mujeres que rechazaron la masturbación o mostraron inconformidad con su sexualidad tuvieron mayor prevalencia de dispareunia, lo cual es acorde con otros autores que citan la actitud negativa de la mujer hacia el sexo como el factor de riesgo más fuerte para el desarrollo de disfunciones sexuales (OR: 19,06; IC95\%: $5,67-64,06)^{28}$.

Los problemas sexuales posparto son muy frecuentes ${ }^{9,10,26}$. En este estudio, de las mujeres sexualmente activas y sin dificultades sexuales antes del parto, casi la mitad refirió algún grado de deterioro de su vida sexual después del parto, lo que sugiere una evidente relación entre el parto y el deterioro de la función sexual; datos acordes con lo expresado en otras publicaciones $^{29,30}$.

Con relación al antecedente de dispareunia, se pudo confirmar la asociación de dicho antecedente con la aparición de dispareunia posparto; se observó que esta se incrementa en el posparto a más del doble, en comparación con las mujeres que no tienen el antecedente. Además, se pudo observar que el porcentaje del antecedente de dispareunia es similar al descrito por otros autores ${ }^{5,31}$. Por otra parte, destaca que las mujeres con dispareunia previa al parto mantenían menos relaciones sexuales semanales posparto (OR: 2,00; IC95\%: 1,05-3,00), justificándose por diferentes motivos que no solo involucraban al dolor coital, ya que son muchos los factores que influyen en la aparición de dispareunia posparto, incluyendo el tipo de entrega ${ }^{2}$, la episiotomía, la lactancia, la presencia de dispareunia antes y durante el embarazo, así como la paridad ${ }^{32}$.

En este estudio se observó que la dispareunia suele afectar a un porcentaje significativo de mujeres después del parto, independientemente de la vía de este. Se hace imperioso reconocer los potenciales factores asociados a su etiología para poder brindarles un apropiado y óptimo manejo durante la atención del parto, y así facilitar la reanudación de la función sexual normal y prevenir la morbilidad física y psicosocial propia de esta complicación.

Se afirma con esta investigación que la dispareunia mantiene la constancia de una disminución y un franco deterioro de la actividad sexual en el posparto, así como de la progresión en la mala relación de pareja.
La evaluación de los trastornos sexuales posparto se hace difícil en nuestro medio, y por eso es de vital relevancia realizar una adecuada valoración psicosexual antes de la atención del parto, y ojalá antes del embarazo (durante la consulta preconcepcional), pues es indiscutible que el perfil psicológico de la mujer tiene una tremenda influencia e importancia en las alteraciones sexuales ${ }^{33}$ durante el puerperio.

Se ha detectado en este estudio que la preservación de la función sexual en el posparto no se ve favorecida por la cesárea, ya que esta resulta ineficaz en la prevención de las alteraciones sexuales posparto; por lo tanto, no estaría recomendada de manera electiva o sistemática en aquellas mujeres sin indicación.

Como fortalezas de este estudio destaca el importante número de participantes y la utilización de una herramienta validada y estandarizada con características psicométricas adecuadas. Una de las limitaciones es que no se pudo recolectar suficiente muestra de la población afiliada al régimen subsidiado, y la selección de la muestra a partir de los códigos CIE-10 podría llevar a sesgos de selección y de información.

\section{Conclusiones}

La prevalencia de dispareunia en el posparto en las mujeres de Armenia sobrepasa la tercera parte de la población. Existe una importante combinación de factores de riesgo potencialmente controlables. El conocimiento de la asociación existente entre la aparición de dispareunia en el posparto con ciertos factores asociados a la conducta del obstetra ayudaría a facilitar la toma de decisiones obstétricas apropiadas. Ante la alta prevalencia de la dispareunia posparto, así como su negativo impacto en la calidad de vida de las mujeres que la padecen, hace imperioso centrar la atención en planificar y diseñar intervenciones efectivas para prevenir la aparición de este catastrófico trastorno.

\section{Agradecimientos}

Se agradece a las mujeres participantes de esta investigación, al personal de enfermería, las trabajadoras sociales, las psicólogas, los bioestadísticos y los epidemiólogos que nos apoyaron. Asimismo, el reconocimiento a la labor de mi padre y maestro, el señor José F. Espitia Hernández, por su permanente apoyo en la revisión y el desarrollo de este proyecto. 


\section{Financiamiento}

Esta investigación fue financiada por recursos propios del autor, que fueron obtenidos de sus turnos de obstetricia.

\section{Conflicto de intereses}

El autor declara no tener conflictos de intereses.

\section{Responsabilidades éticas}

Protección de personas y animales. Los autores declaran que para esta investigación no se han realizado experimentos en seres humanos ni en animales.

Confidencialidad de los datos. Los autores declaran que han seguido los protocolos de su centro de trabajo sobre la publicación de datos de pacientes.

Derecho a la privacidad y consentimiento informado. Los autores declaran que en este artículo no aparecen datos de pacientes.

\section{Bibliografía}

1. Lewis RW, Fugl-Meyer KS, Corona G, Hayes RD, Laumann EO Moreira ED Jr, et al. Definitions/epidemiology/risk factors for sexual dysfunction. J Sex Med. 2010; 7:1598-607.

2. O'Malley D, Higgins A, Begley C, Daly D, Smith V. Prevalence of and risk factors associated with sexual health issues in primiparous women at 6 and 12 months postpartum; a longitudinal prospective cohort study (the MAMMI study). BMC Pregnancy Childbirth. 2018;18:196.

3. Tayyeb M, Gupta V. Dyspareunia. StatPearls. Treasure Island (FL): StatPearls Publishing; June 11, 2021

4. Espitia De La Hoz FJ. Prevalencia de disfunción sexual en mujeres de Eje Cafetero, Rev Cienc Biomed. 2016;7:25-33.

5. Danielsson I, Sjöberg I, Stenlund H, Wikman M. Prevalence and incidence of prolonged and severe dyspareunia in women: results from a population study. Scand J Public Health. 2003;31:113-8.

6. Eid MA, Sayed A, Abdel-Rehim R, Mostafa T. Impact of the mode of delivery on female sexual function after childbirth. Int J Impot Res. 2015;27:118-20.

7. Espitia De La Hoz FJ. Evaluación de la prevalencia de disfunción sexua en mujeres médicos, del Eje Cafetero colombiano, en etapa de climaterio. Arch Med (Col). 2017;17:70-7.

8. Fergus KB, Cohen AJ, Cedars BE, Rowen TS, Patino G, Breyer BN. Risk factors for sexual pain among physically active women. Sex Med. 2020:8:501-9.

9. Manresa M, Pereda A, Bataller E, Terre-Rull C, Ismail KM, Webb SS Incidence of perineal pain and dyspareunia following spontaneous vaginal birth: a systematic review and meta-analysis. Int Urogynecol J. 2019;30:853-68

10. Banaei M, Kariman N, Ozgoli G, Nasiri M, Ghasemi V, Khiabani A, et al. Prevalence of postpartum dyspareunia: a systematic review and meta-analysis. Int J Gynaecol Obstet. 2021;153:14-24.
11. Espitia FJ. Mi primer año en La Crónica. Bogotá: Editorial Bolívar. 2016 p. $111-2$.

12. Isidori AM, Pozza C, Esposito K, Giugliano D, Morano S, Vignozzi L, et al. Development and validation of a 6 -item version of the female sexual function index (FSFI) as a diagnostic tool for female sexual dysfunction. J Sex Med. 2010;7:1139-46.

13. Rosen R, Brown C, Heiman J, Leiblum S, Meston C, Shabsigh R, et al. The Female Sexual Function Index (FSFI): a multidimensional self-report instrument for the assessment of female sexual function. J Sex Marital Ther. 2000;26:191-208.

14. Chedraui $P$, Pérez-López FR, Sánchez $H$, Aguirre $W$, Martínez $N$, Miranda $\mathrm{O}$, et al. Assessment of sexual function of mid-aged Ecuadorian women with the 6-item Female Sexual Function Index. Maturitas. 2012;71:407-12.

15. Abdool Z, Thakar R, Sultan $A H$. Postpartum female sexual function. Eur J Obstet Gynecol Reprod Biol. 2009;145:133-7.

16. Alligood-Percoco NR, Kjerulff KH, Repke JT. Risk factors for dyspareunia after first childbirth. Obstet Gynecol. 2016;128:512-8.

17. Alp Yilmaz F, Avci D, Arzu Aba Y, Ozdilek R, Dutucu N. Sexual dysfunction in postpartum Turkish women: it's relationship with depression and some risk factors. Afr J Reprod Health. 2018:22:54-63.

18. Mitchell KR, Geary R, Graham CA, Datta J, Wellings K, Sonnenberg P, et al. Painful sex (dyspareunia) in women: prevalence and associated factors in a British population probability survey. BJOG. 2017:124:1689-97.

19. Barrett G, Pendry E, Peacock J, Victor C, Thakar R, Manyonda I. Women's sexual health after childbirth. BJOG. 2000;107:186-95.

20. McDonald EA, Gartland D, Small R, Brown SJ. Dyspareunia and childbirth: a prospective cohort study. BJOG. 2015;122:672-9.

21. Buhling KJ, Schmidt S, Robinson JN, Klapp C, Siebert G, Dudenhausen JW. Rate of dyspareunia after delivery in primiparae according to mode of delivery. Eur J Obstet Gynecol Reprod Biol. 2006; $124: 42-6$.

22. Manresa M, Pereda A, Goberna-Tricas J, Webb S, Terre-Rull C Bataller E. Postpartum perineal pain and dyspareunia related to each superficial perineal muscle injury: a cohort study. Int Urogynecol J. 2020;31:2367-75.

23. Latthe P, Mignini L, Gray R, Hills R, Khan K. Factors predisposing women to chronic pelvic pain: systematic review. BMJ. 2006;332:749-55.

24. Savoy M, O'Gurek D, Brown-James A. Sexual health history: techniques and tips. Am Fam Physician. 2020;101:286-93.

25. Jaafarpour M, Khani A, Khajavikhan J, Suhrabi Z. Female sexual dysfunction: prevalence and risk factors. J Clin Diagn Res. 2013;7:2877-80.

26. Rosen NO, Pukall C. Comparing the Prevalence, Risk Factors, and Repercussions of Postpartum Genito-Pelvic Pain and Dyspareunia. Sex Med Rev. 2016;4:126-35.

27. Sobhgol SS, Priddis H, Smith CA, Dahlen HG. The effect of pelvic floor muscle exercise on female sexual function during pregnancy and postpartum: a systematic review. Sex Med Rev. 2019;7:13-28.

28. Song $\mathrm{SH}$, Jeon $\mathrm{H}$, Kim SW, Paick JS, Son H. The prevalence and risk factors of female sexual dysfunction in young Korean women: an internet-based survey. J Sex Med. 2008:5:1694-701.

29. Maamri A, Badri T, Boujemla H, El Kissi Y. Sexuality during the postpartum period: study of a population of Tunisian women. Tunis Med. 2019;:97:704-10.

30. Dağli E, Kul Uçtu A, Özerdoğan N. Sexual dysfunction in the postpartum period: its relationship with postpartum depression and certain other factors. Perspect Psychiatr Care. 2021;57:604-9.

31. Hill DA, Taylor CA. Dyspareunia in women. Am Fam Physician. 2021;103:597-604

32. Lagaert L, Weyers S, Van Kerrebroeck H, Elaut E. Postpartum dyspareunia and sexual functioning: a prospective cohort study. Eur $\mathrm{J}$ Contracept Reprod Health Care. 2017;22:200-6.

33. Espitia-De La Hoz FJ. Prevalence and characterisation of sexual dysfunctions in women, in 12 Colombian cities, 2009-2016. Rev Colomb Obstet Ginec. 2018;69:9-21. 